\title{
Heat Transfer inside a Highway Tunnel under Vehicle Thermal Effect
}

\author{
Tao Zhang, Hiroshi Katsuchi, Hitoshi Yamada \\ Yokohama National University \\ 79-1 Tokiwadai, Hodogaya-ku, Yokohama 240-8501, Japan \\ zhang-tao-nr@ynu.jp; katsuchi@ynu.ac.jp; yamada-hitoshi-cj@ynu.ac.jp
}

\begin{abstract}
A highway tunnel in Tokyo experiences high inside temperature, especially during summer season. Vehicle heat was roughly calculated based on fuel consumption of different vehicle under observed traffic volume. In a certain range of tunnel, the amount of heat absorbed by road surface and concrete wall, extracted by ventilation system and went out by tunnel exit, were calculated respectively in short time period. These results are used to verify a heat-balance model to determine each proportion of vehicle heat dissipated in road, concrete wall and outside air. Finally, cooling measure based on this is preliminary discussed.
\end{abstract}

Keywords: Road Tunnel, Vehicle Heat, Ventilation, Concrete Wall, Heat Balance.

\section{Introduction}

A road tunnel in Tokyo is too hot inside during summer, the highest air temperature can be up to $48.3^{\circ} \mathrm{C}$ at midday, which is obviously over normal level for motorcyclist and sometimes even hard for vehicle air condition to operate normally. Generally, in case of short and medium road tunnel, temperature rising problem is not considered because of its limited traffic volume, and heat generated by running vehicle can be easily dissipated either into surrounding earth or outside environment via exit. Therefore, pollutant extraction is mainly focused on to ensure air quality inside. However, for long tunnel with high traffic in hot season, especially during congestion time, temperature rising issue cannot be ignored. Actually, because of bad heat dissipation inside tunnel, temperature increase along tunnel axial direction along with traffic induced wind. In previous research [1,2], heat transfer in underground railway system was studied, as a result, periodical passing train generated the most heat load inside tunnel, heat balance model and several parameters were determined afterwards.

In this study, more complicated road traffic heat is studied. Temperature distribution of road surface and concrete wall inside are measured in several spots along tunnel, traffic volume and ventilation operation details are also collected. 2 types of vehicle, representing general types on road, are measured by field test: medium truck [3], passenger car [4]. These results are used to build a heat-balance model [5], and verified using real measurement data. Finally, cooling measure is discussed based on the model.

\section{Heat Transfer Model inside the Tunnel}

A model has been made to evaluate heat transfer inside the road tunnel system, including all main possible way the heat energy can transfer. A 1690m long with typical shield cross-section range is selected as calculation objects, moisture, traffic volume and temperature sensors are applied on both side. Based on measurement data inside, the air relative humidity (RH) is always around $48 \%$ without large variation, it only absorbs $0.2 \%$ heat compared with air inside tunnel if they both increased $1^{\circ} \mathrm{C}$, which means the latent energy of water vapor can be neglected. All physical properties used in this study are provided in table 1.

The model is made and calculated using Excel and Matlab to deal with several groups of data. All heat energy mentioned above shall be balanced as

$$
\mathrm{Q}_{\text {air }}+Q_{\text {road }}+Q_{\text {wall }}+\mathrm{Q}_{\text {ventilation }}+\mathrm{Q}_{\text {outlet }}=Q_{\text {vehicle }}
$$

In Fig. 1, vehicle generated heat and other parts are demonstrated. Inlet is on $19.89 \mathrm{kp}$ ( $\mathrm{km}$ position), and outlet on $21.58 \mathrm{kp}$. Traffic direction wind velocity is induced by traffic flow. 


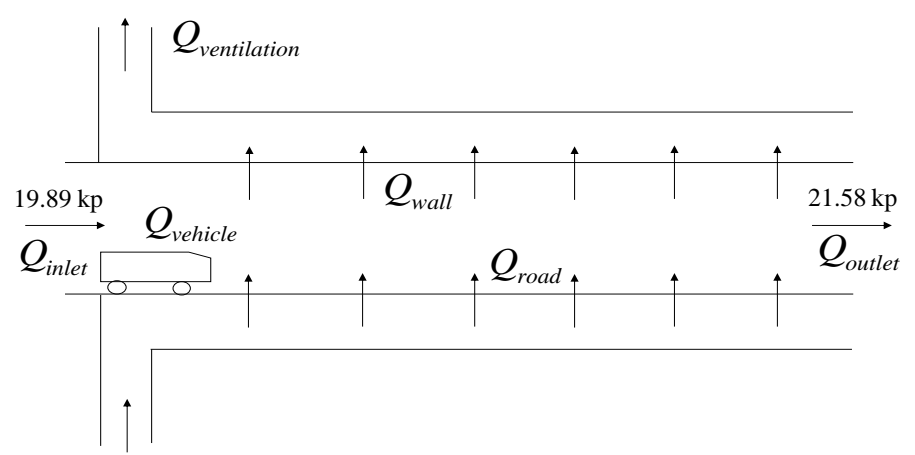

Fig. 1: Model of heat load in tunnel.

\subsection{Vehicle Generated Heat}

As previously mentioned, the thermal effects of vehicles are originally from fuel combustion, which varies significantly due to many random factors. The vehicle heat is the mainly cause for high temperature, in fact when vehicle runs in tunnel, the burning of fossil fuel generates a huge amount of energy which is extremely hard to quantify both in time and special domain (along driving direction) as too many random factors exist there, such as driving behaviour, road gradient [6], vehicle types [7], traffic flow velocity, air-condition in vehicle, etc. However, most (around 60\%) of total energy [8] goes to air inside tunnel directly as hot exhaust gas around $260^{\circ} \mathrm{C}$ [9], the temperature of exhaust pipe can also reach at $210^{\circ} \mathrm{C}$. The rest (around $40 \%$ ) becomes mechanical energy [10] which finally also converts into heat due to friction loss, aerodynamic drag, vehicle internal friction, etc.

Here we don't focus on calculate the details about each parts of vehicle energy consumption, the total heat given out by traffic flow is calculated based on fuel consumption for small and medium vehicle (length less than $5.5 \mathrm{~m}$, mainly consume gasoline $12 \mathrm{~km} / \mathrm{L}$ ) and large vehicle (longer than $5.5 \mathrm{~m}$, mainly with diesel engine, consume $5 \mathrm{~km} / \mathrm{L}$ ). Assume that during congestion fossil fuel consumes 3 times than normal speed when running on same distance. Heat value of gasoline and diesel are $32 \mathrm{MJ} / \mathrm{L}$ and $39 \mathrm{MJ} / \mathrm{L}$. Heat absorption by vehicle is neglected. Data analysis based on these assumptions is conducted to predict a time-averaged heat flux given out by traffic flow as shown in Fig. 2.

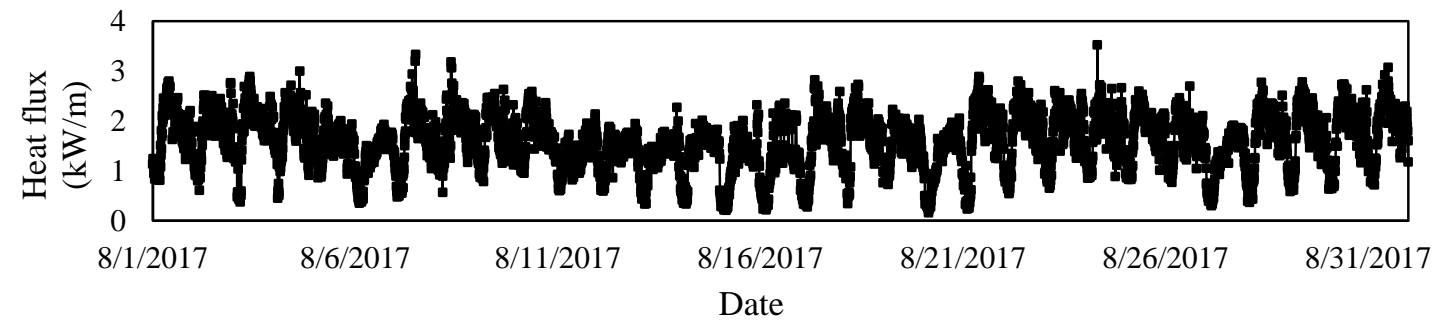

Fig. 2: Estimated vehicle heat flux between $19.89 \mathrm{kp}$ and $21.58 \mathrm{kp}$.

\subsection{Inlet and Outlet}

This model is made from only one part of tunnel, so there is axial air movement due to traffic effect. It can be known from data that Temperature in outlet spot is higher than inlet, suppose air is incompressible and air mass flow rate is same on both sides, then we have

$$
\mathrm{Q}_{\text {outlet }}=c_{\text {air }} \rho_{\text {air }} v_{\text {outlet }} S\left(T_{\text {outlet }}-T_{\text {inlet }}\right) \quad V_{\text {outlet }}=\mathrm{S} v_{\text {inlet }}+V_{\text {vinet }}-V_{\text {voutlet }}
$$

$\rho_{\text {air }}$ and $c_{\text {air }}$ represent density and heat capacity of air. Inlet and outlet air flow velocity, temperature in traffic direction are denoted as $v_{\text {inlet }}$ and $v_{\text {outlet }}, T_{\text {inlet }}$ and $T_{\text {outlet }}$. Air flow amount due to inlet and outlet of ventilation system are defined as $V_{\text {vinet }}, V_{\text {voutlet }}$. Tunnel cross section area $\mathrm{S}$ is $63.3 \mathrm{~m}^{2}$. 


\subsection{Ventilation}

The ventilation of road tunnel is to control the pollutant concentration and visibility in tunnel effectively, and exchanging heat with ambient air. The calculation length $1690 \mathrm{~m}$ is subjected to transverse ventilation system, heat extracted by ventilation can be calculated by

$$
\begin{gathered}
\mathrm{Q}_{\text {ventilation }}=c_{\text {air }} \rho_{\text {air }} \Delta V_{\text {ventilation }}\left(T_{\text {outlet }}-T_{a}\right) \\
c_{\text {air }} \rho_{\text {air }}\left(v_{\text {inlet }} S+V_{\text {vinet }}\right) T_{a}=c_{\text {air }} \rho_{\text {air }} V_{\text {vinlet }} T_{\text {vinlet }}+c_{\text {air }} \rho_{\text {air }} v_{\text {vinlet }} S T_{\text {inlet }}
\end{gathered}
$$

$T_{a}$ is average air temperature inside tunnel, $T_{\text {vinlet }}$ and $T_{\text {voutlet }}$ are inlet and outlet air temperature of ventilation system.

\subsection{Asphalt Road Surface}

Road surface absorbs a limited part of heat from vehicle, Prusa et al. (2002) [11] showed the quasi-steady energy balance in a certain range near the vehicle due to heat generated by itself, and evaluated the thermal fluxes generated by vehicles such as the surface thermal flux under the effect of frictional loss, radiant flux at the bottom, convective heat transfer that occurs between vehicle and the road surface.

Heat conductivity $k$ has been calculated by data from wind-tunnel test as $0.89 \mathrm{~W} / \mathrm{m} \cdot{ }^{\circ} \mathrm{C}$, density is also determined after measuring the asphalt test specimen. In this study, complex heat exchange between road pavement and air inside tunnel will not be conducted. Instead, field measurement data has been used. Heat absorbed is calculated using

$$
Q_{\text {road }}=k \frac{\partial \mathrm{T}_{a s}}{\partial \mathrm{d}} \mathrm{W} \Delta t+0.75 \mathrm{DW} c_{a s} \rho_{a s} \Delta T_{a s}
$$

$\mathrm{T}_{a s}$ and $\mathrm{d}$ are asphalt temperature and depth. $\mathrm{W}$ is road width. $\mathrm{D}$ is total depth of asphalt pavement. $c_{a s}$ and $\rho_{a s}$ are heat capacity and density respectively.

\subsection{Wall Absorption}

Heat absorbed by wall is mainly by convective heat transfer between air and wall. The convective coefficient $h_{c}$ is estimated by using Colburn correlation as below

$$
\mathrm{Nu}=0.023 \operatorname{Re}^{0.8} \operatorname{Pr}^{1 / 3} \quad h_{c}=\frac{\lambda}{\mathrm{d}} \mathrm{Nu} \quad Q_{\text {wall }}=h_{c} C\left(T_{a}-T_{w}\right) \Delta t
$$

$\mathrm{Nu}$ is Nusselt Number, Re is Reynolds Number of air, Pr is Prandtl Number of air. $h_{c}$ represents convective heat transfer coefficient. $\mathrm{C}$ is concrete wall perimeter. $T_{w}$ is concrete wall temperature.

Table 1: Physical properties.

\begin{tabular}{|c|c|c|c|}
\hline Air density $\rho_{\text {air }}$ & $1.127 \mathrm{~kg} / \mathrm{m}^{3}$ & Radius of tunnel R & $5.55 \mathrm{~m}$ \\
\hline Specific heat of air $c_{\text {air }}$ & $1.005 \mathrm{~kJ} / \mathrm{kg} \cdot{ }^{\circ} \mathrm{C}$ & Calculation length of tunnel L & $1690 \mathrm{~m}$ \\
\hline Asphalt density $\rho_{\text {as }}$ & $2360 \mathrm{~kg} / \mathrm{m}^{3}$ & Cross section area S & $63.3 \mathrm{~m}^{2}$ \\
\hline Asphalt heat conductivity k & $0.89 \mathrm{~W} / \mathrm{m}^{\circ} \mathrm{C}$ & Concrete wall perimeter C & $23.25 \mathrm{~m}$ \\
\hline Asphalt thickness d & $0.08 \mathrm{~m}$ & Road width W & $7.5 \mathrm{~m}$ \\
\hline Asphalt heat capacity $c_{a s}$ & $0.92 \mathrm{~kJ} / \mathrm{kg} \cdot{ }^{\circ} \mathrm{C}$ & Heat conductivity of air & $0.0271 \mathrm{~W} / \mathrm{m} \cdot{ }^{\circ} \mathrm{C}$ \\
\hline Prandtl Number of air Pr & 0.7 & \\
\hline \multicolumn{4}{|l|}{} \\
\hline Inlet and outlet air flow velocity & $v_{\text {inlet }} v_{\text {outlet }}$ & Ventilation outlet air volume & $V_{\text {voutlet }}$ \\
\hline Average air temperature & $T_{a}$ & Ventilation inlet air volume & $V_{\text {vinlet }}$ \\
\hline Inlet and outlet air temperature & $T_{\text {inlet }} T_{\text {outlet }}$ & Ventilation inlet and outlet air & $T_{\text {vinlet }} T_{\text {voutlet }}$ \\
\hline Convective heat transfer coefficient & $h_{c}$ & Wall temperature & $T_{a}$ \\
\hline
\end{tabular}




\section{Validation and Suggestion}

Based on the results above, the predicted inside air temperature at $21.58 \mathrm{kp}$ location is compared with real measurement data, it turns out to be the predicted air temperature variation is too large, although total energy is almost balanced in one day. This model needs to be made much more detailed in order to predict air temperature more accurately. In Fig. 3, it shows that each heat load changes during time in one day, ventilation extracted heat is more than other 3 kinds, which means most of traffic induced heat dissipates with air flow forced by ventilation system in transverse direction. To reduce temperature, compared with applying good thermal conductivity materials on wall or water-retaining asphalt on road, continuous air cooling methods are better to be adopted. For example, water mist spray inside tunnel is an economic and effective way to consider.

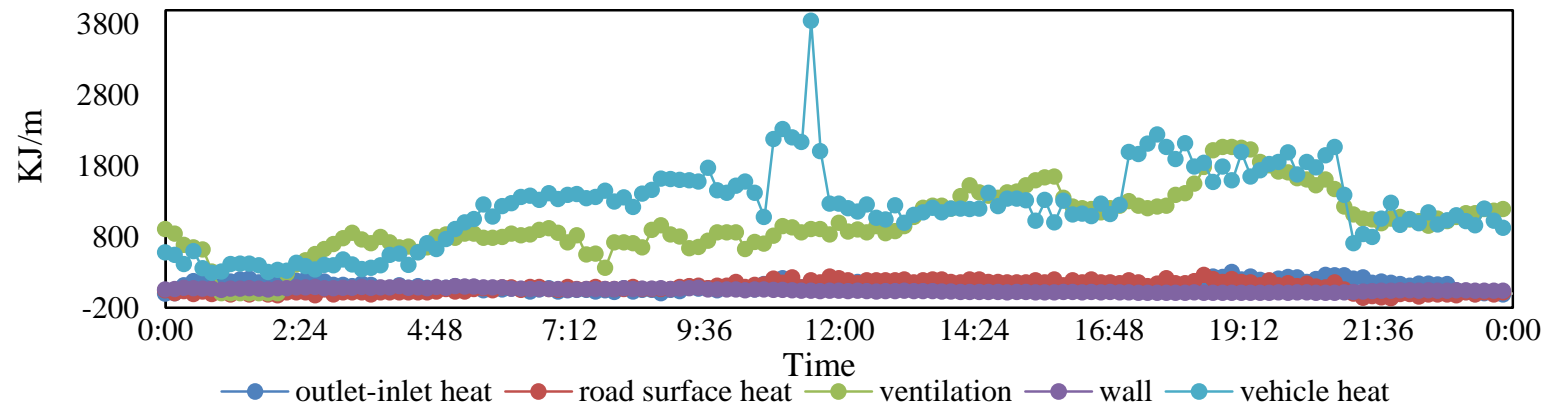

Fig. 3: Total heat gain and loss in $7^{\text {th }}$, Aug.

\section{Conclusion}

The heat investigation based on measurement data in a long highway tunnel has been performed. It shows that vehicle heat is the main heat source that cause temperature rising. Most vehicle heat energy is extracted out by air flow inside tunnel. Cooling countermeasures must be concentrate on continuous air cooling methods as air flow carries most heat energy heated by running vehicle.

\section{References}

[1] F. Ampofo, G. Maidment, and J. Missenden, "Underground railway environment in the UK Part 2: Investigation of heat load," Applied Thermal Engineering, vol. 24, no. 5, pp. 633-645, 2004.

[2] S. Sadokierski, J.-L. Thiffeault, Heat transfer in underground rail tunnels. arXiv preprint, arXiv:0709.1748, 2007.

[3] K. Holmberg, et al., "Global energy consumption due to friction in trucks and buses," Tribology International, vol. 78, pp. 94-114, 2014.

[4] K. Liu, et al., "Modelling the multilevel structure and mixed effects of the factors influencing the energy consumption of electric vehicles," Applied Energy, vol. 183, pp. 1351-1360, 2016.

[5] A. Fujimoto, A. Saida, and T. Fukuhara, "A New Approach to Modeling Vehicle-Induced Heat and Its Thermal Effects on Road Surface Temperature," Journal of Applied Meteorology and Climatology, vol. 51, no. 11, pp. 19801993, 2012.

[6] S Carrese, A. Gemma, and S. La Spada, "Impacts of driving behaviours, slope and vehicle load factor on bus fuel consumption and emissions: a real case study in the city of Rome," Procedia-Social and Behavioral Sciences, vol. 87, pp. 211-221, 2013.

[7] M. Ziyadi, et al., "Vehicle Energy Consumption and an Environmental Impact Calculation Model for the Transportation Infrastructure Systems," Journal of Cleaner Production, 2017.

[8] N. Kobayashi, and T. Ikegami, "Automobile Industry's action on Environments and Relevant Thermal and Fluid Dynamics," Thermal Science and Engineering, vol. 15, pp. 49-54, 2008.

[9] R. H. Gonzales, and M. Engineer, Diesel exhaust emission system temperature test. US National Technology \& Development Program, 2008. 
[10] B. Hay, et al., The Contribution Of The Metrology Of Thermophysical Properties Of Materials In Automotive Design.

[11] J. M. Prusa, et al., "Conceptual and scaling evaluation of vehicle traffic thermal effects on snow/ice-covered roads," Journal of Applied Meteorology, vol. 41. no. 12, pp. 1225-1240, 2002. 